\title{
Traction Control Method of Hybrid Electric Vehicle based on Multi-Objective Dynamic Coordination Control
}

\author{
Feng Zhao \\ Yugong Luo \\ zhaofeng2222@126.com_lyg@tsinghua.edu.cn \\ Keqiang Li \\ likq@tsinghua.edu.cn \\ State Key Laboratory of Automotive Safety and Energy, Tsinghua University, Beijing 100084, P.R. China
}

\begin{abstract}
The control method of the conventional traction control system on split- $\mu$ surfaces improves vehicle acceleration performance, but influences its stability performance. To solve this problem, a hierarchical traction control system for ISG hybrid electric vehicles based on multi-objective dynamic coordination control (MHEVTCS) is proposed. In the upper level controller, a target driving torque calculating strategy based on dynamical sliding mode control is developed. In the lower level controller, such strategies as multi-objective dynamic coordination control strategy, brake torque control strategy based on an inverse model, target engine torque design strategy and torque dynamic coordinate control strategy are proposed. Detailed simulation and hardware-in-loop experiment results show that slipping wheels are controlled quickly, accurately and smoothly by MEHVTCS. MHEVTCS solves the problem of merely pursuing acceleration performance and neglecting stability performance of conventional traction control system.
\end{abstract}

Keywords: Hybrid Electric Vehicle, Traction Control System, Coordination Control

\section{Introduction}

On split- $\mu$ surfaces, traction control system of conventional internal combustion engine vehicle (ICETCS) applies brake torque on the slipping wheel of low adhesion side road to improve vehicle's acceleration performance $^{[1][2]}$. However, additional yaw moment is caused which influences its stability performance.

Motor system of hybrid electric vehicle (HEV) has better dynamic performance than engine system and hydraulic system ${ }^{[3][4]}$. It can provide a large drive torque at a low speed. Compared to conventional internal combustion engine vehicle, HEV is easier to slip on slippery road ${ }^{[5][6]}$. If the control method of ICETCS on split- $\mu$ surfaces is continually used by HEV, more brake torque intervening should be used to maintain the slip ratio of slipping wheel at a desired value. And, more additional yaw moment will be caused that influences vehicle's stability severely. Thus, 
traction control system of HEV (HEVTCS) should be redesigned.

Taking an ISG hybrid electric vehicle as the research object as shown in Figure1, the traction control method of an ISG hybrid electric vehicle is researched in this paper.

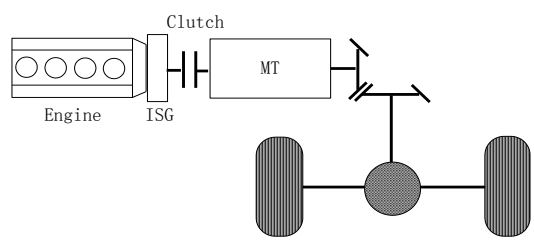

Figure1: Configuration of the research object

\section{Hierarchical Control System}

Based on a typical structure of HEVTCS, MEHVTCS is proposed as shown in Figure2 which is composed of two level controllers.

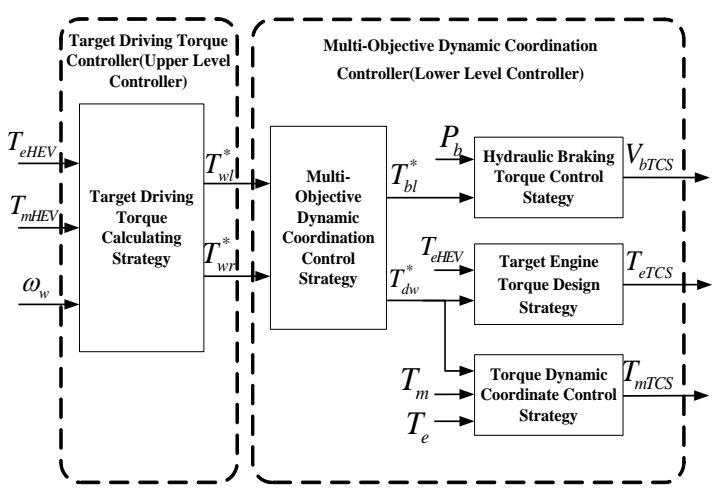

Figure2: Control structure of MEHVTCS

In the upper level controller, a target driving torque calculating strategy determines target driving torques exerted on left front wheel $T_{w l}^{*}$ and exerted on right front wheel $T_{w r}^{*}$ according to the desired engine torque $T_{\text {eHEV }}$, the desired motor torque $T_{m H E V}$ and the wheel rotation speed $\omega_{w}$ to ensure that slip ratios of all slipping wheels can be maintained at desired values. In the lower level controller, several strategies such as multi-objective dynamic coordination control strategy, brake torque control strategy based on an inverse model, target engine torque design strategy and torque dynamic coordinate control strategy are proposed to control the engine torque demand $T_{\text {eтCS }}$, the motor torque demand $T_{m T C S}$ and the solenoid valve opening demand $V_{b T C S}$ in coordination to track the target driving torques $T_{w l}^{*}$ and $T_{w r}^{*}$ which are calculated by the upper level controller. In Figure2, $T_{\text {eHEV }}$ and $T_{\text {mHEV }}$ are calculated by the energy management system, $T_{b l}^{*}$ is the brake torque demand exerted on the low adhesion wheel, $T_{d w}^{*}$ is the driving torque demand exerted on the wheels, $P_{b}$ is the actual brake pressure, $T_{e}$ is the actual engine torque, $T_{m}$ is the actual motor torque.

\section{Target Driving Torque}

\section{Control for the Upper Level}

\section{Controller}

In the target driving torque calculating strategy, a dynamical sliding mode controller of multi-input and multi-output is designed as shown in Figure3.

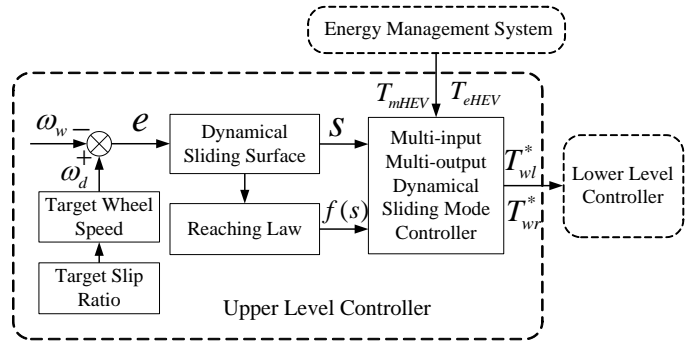

Figure3: Target driving torque control strategy

Taking the whole vehicle model as the control system

$\left\{\begin{array}{l}\dot{x}_{1}=a_{11} x_{1}^{2}+a_{12} f_{1}\left(x_{1}, x_{2}\right)+a_{13} f_{2}\left(x_{1}, x_{3}\right) \\ \dot{x}_{2}=a_{21} f_{1}\left(x_{1}, x_{2}\right)+a_{22} u_{1} \\ \dot{x}_{3}=a_{31} f_{2}\left(x_{1}, x_{3}\right)+a_{32} u_{2}\end{array}\right.$

where $x_{1}=v_{x}, \quad x_{2}=\omega_{f l}, \quad x_{3}=\omega_{f r}, \quad u_{1}=T_{w l}^{*}$, $u_{2}=T_{w r}^{*} \quad, \quad \mu_{l}\left(v_{x}, \omega_{f l}\right)=f_{1}\left(x_{1}, x_{2}\right) \quad$, $\mu_{r}\left(v_{x}, \omega_{f r}\right)=f_{2}\left(x_{1}, x_{3}\right), \quad a_{11}=-\rho A C / 2 m$, 
$a_{12}=a_{13}=\gamma_{f} g / 2, \quad a_{21}=a_{31}=-\gamma_{f} m g r_{\omega} / 2 J_{\omega}$, $a_{22}=a_{32}=1 / J_{\omega}, m$ is the vehicle mass, $v_{x}$ is the vehicle longitudinal speed, $J_{\omega}$ is the inertia of the wheel assembly, $r_{\omega}$ is the wheel radius, $\rho$ is the air density, $A$ is the frontal area, $C$ is the air resistance coefficient, $\gamma_{f}$ is the front axle load proportion.

The control target is to make $x_{2}$ and $x_{3}$ to follow $n x_{1}$ together as

$\left\{\begin{array}{l}x_{2}=n x_{1} \\ x_{3}=n x_{1}\end{array}\right.$

where $n=1 / r_{\omega}\left(1-\lambda_{d}\right), \quad \lambda_{d}$ is the optimal slip ratio.

The switching surface are

$\left\{\begin{array}{l}s_{1}=\left(x_{2}-n x_{1}\right)^{\prime}+c_{1}\left(x_{2}-n x_{1}\right)+d_{1} u_{1} \\ s_{2}=\left(x_{3}-n x_{1}\right)^{\prime}+c_{2}\left(x_{3}-n x_{1}\right)+d_{2} u_{2}\end{array}\right.$

where $c_{1}$ and $c_{2}$ are fixed positive constants, $d_{1}$ and $d_{2}$ are controller parameters, and $d_{1}, d_{2}>0$.

The reaching law is

$$
\left\{\begin{array}{l}
\dot{s}_{1}=f\left(s_{1}\right)=-k_{1} s_{1}-\varepsilon_{1} \operatorname{sgn}\left(s_{1}\right) \\
\dot{s}_{2}=f\left(s_{2}\right)=-k_{2} s_{2}-\varepsilon_{2} \operatorname{sgn}\left(s_{2}\right)
\end{array}\right.
$$

where $k_{1}, k_{2}, \varepsilon_{1}$ and $\varepsilon_{2}$ are positive.

Then the control law is

$$
\left\{\begin{array}{l}
\dot{u}_{1}=\frac{1}{d_{1}}\left[-\left(x_{2}-n x_{1}\right)^{\prime \prime}-c_{1}\left(x_{2}-n x_{1}\right)^{\prime}+f\left(s_{1}\right)\right] \\
\dot{u}_{2}=\frac{1}{d_{2}}\left[-\left(x_{3}-n x_{1}\right)^{\prime \prime}-c_{2}\left(x_{3}-n x_{1}\right)^{\prime}+f\left(s_{2}\right)\right]
\end{array}\right.
$$

\section{Multi-Objective Dynamic}

Coordination Control for the

\section{Lower Level Controller}

\subsection{Multi-Objective Dynamic Coordination Control Strategy}

As described in the introduction, on split- $\mu$ surfaces, MHEVTCS can not merely pursue maximum of vehicle's acceleration performance like ICETCS. It should take advantage of the adhesion condition of high adhesion side road to improve vehicle's acceleration performance without influencing its stability severely. According to this idea, a multi-objective dynamic coordination control strategy is proposed.

There are two control objectives in this strategy. The first objective is to satisfy vehicle's stability performance by decreasing hydraulic brake torque exerted on the low adhesion wheel as in

$\min T_{b l}^{*}$

The second objective is to improve vehicle's dynamic performance by increasing driving torque exerted on the wheels as in

$\max T_{d w}^{*}$

According to the wheels rotation dynamics and the control target of MHEVTCS which is to make the rotation speed of low adhesion wheel around the desired value and to ensure that high adhesion wheel dose not slip, the inequalities for constraints are

$\left\{\begin{array}{l}0.9 T_{w l}^{*} \leq T_{d w}^{*}-T_{b l}^{*} \leq 1.1 T_{w l}^{*} \\ T_{d w}^{*} \leq T_{w h}^{*}\end{array}\right.$

Merging these two control objectives together, this strategy can be described as one constrained multi-objective optimization problem, as shown in

$$
\min \left[T_{b l}^{*},-T_{d w}^{*}\right]
$$

s.t.:

$\left\{\begin{array}{l}0.9 T_{w l}^{*} \leq T_{d w}^{*}-T_{b l}^{*} \leq 1.1 T_{w l}^{*} \\ 0<T_{d w}^{*} \leq\left(T_{e \max }+T_{m \max }\right) i_{g} i_{0} \\ T_{d w}^{*} \leq T_{w h}^{*} \\ 0 \leq T_{b l}^{*} \leq T_{w h}^{*}-0.9 T_{w l}^{*}\end{array}\right.$

where $T_{e \max }$ is the maximum engine torque, $T_{m \text { max }}$ is the maximum motor torque, $i_{g}$ is the gear ratio, $i_{0}$ is the final drive ratio.

Hierarchical optimization method is used to solve this multi-objective optimization problem as the following 
1) When the road adhesion coefficient is low or the vehicle speed is high, vehicle's stability performance should be focused on. Then the multi-objective optimization problem can be described as

$\min T_{b l}^{*}$

$\max T_{d w}^{*}$

2) When the road adhesion coefficient is high and the vehicle speed is low, vehicle's dynamic performance should be focused on. At this time, the multi-objective optimization problem can be described as

$\max T_{d w}^{*}$

$\min T_{b l}^{*}$

3) In the transient process, a fuzzy weighting $\xi \in[0,1]$ is defined to turn the multi-objective optimization problem as

$\min \xi T_{b l}^{*}-(1-\xi) T_{d w}^{*}$

s.t.:

$\left\{\begin{array}{l}0.9 T_{w l}^{*} \leq T_{d w}^{*}-T_{b l}^{*} \leq 1.1 T_{w l}^{*} \\ 0<T_{d w}^{*} \leq\left(T_{e \max }+T_{m \max }\right) i_{g} i_{0} \\ T_{d w}^{*} \leq T_{w h}^{*} \\ 0 \leq T_{b l}^{*} \leq T_{w h}^{*}-0.9 T_{w l}^{*}\end{array}\right.$

With the maximum road adhesion coefficient $\mu_{\max }$ and the vehicle speed $v_{e}$ are set as the input of the fuzzy function, the parameter output of the fuzzy adaptor $\xi$ is shown in Figure4.

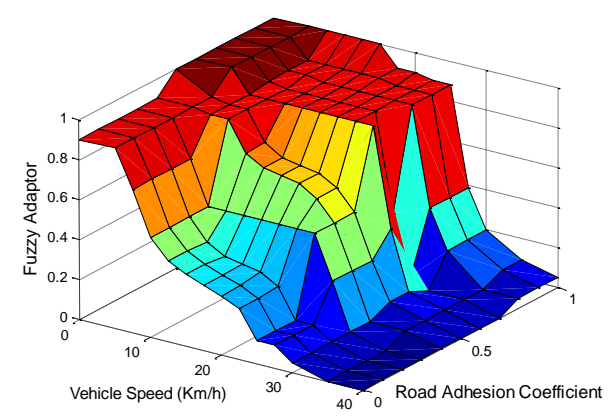

Figure4: Membership function

\subsection{Brake Torque Control Strategy based on an Inverse Model}

The brake torque demand $T_{b l}^{*}$ should be converted to solenoid valve's opening demand, because the hydraulic brake unit can not achieve it directly. Therefore a hydraulic brake torque control strategy based on an inverse model is designed as shown in Figure5.

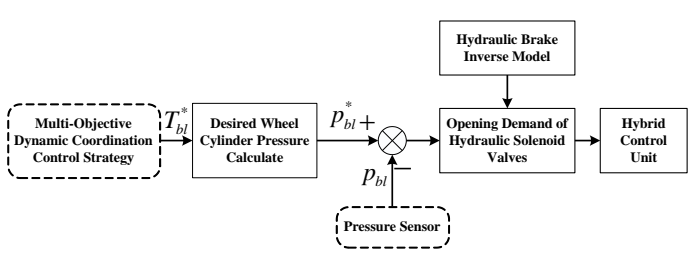

Figure5: Hydraulic brake torque control strategy

Firstly, the brake torque demand $T_{b l}^{*}$ is converted to the desired wheel cylinder pressure $p_{b l}^{*}$ according to friction coefficient of the brake system which is measured by tests. Then, solenoid valve's opening demand is picked up on the basis of an inverse model of wheel cylinder pressure adjusting mechanism as shown in Formula 5 and the differences between the desired wheel cylinder pressure $p_{b l}^{*}$ and the actual wheel cylinder pressure $p_{b l}$.

$$
\begin{aligned}
& t_{a}=\frac{\max \left(p_{b l}^{*}-p_{b l}, 0\right)}{B_{1}\left(p_{a c c}-p_{b l}\right)^{d_{1}}} \\
& t_{d}=\frac{\max \left(p_{b l}-p_{b l}^{*}, 0\right)}{B_{2} p_{b l}{ }^{d_{2}}}
\end{aligned}
$$

\subsection{Target Engine Torque Design Strategy}

After getting driving torque demand exerted on the wheels $T_{d w}^{*}$ in the multi-objective dynamic coordination control strategy, the desired driving total torque $T_{d}^{*}$ which is provided by engine and motor can be calculated

$$
T_{d}^{*}=T_{d w}^{*} / i_{g} / i_{0} / \eta
$$

For HEV, engine is the main power supply. The most effective method to avoid wheels slipping of HEV is to reduce engine torque, but its response speed and control accuracy are worse than motor. Therefore a target 
engine torque design strategy based on low pass filtering is proposed. In this strategy, low frequency part of the desired driving torque is provided by engine and high frequency part of the desired driving torque is compensated by motor. The filtering method is

$$
\begin{aligned}
& T_{d c}^{*}(k)=T_{d c}^{*}(k-1)+ \\
& {\left[T_{s c}^{*}(k)-T_{d c}^{*}(k-1)\right] \frac{T_{s}}{T_{f}}\left(1-\frac{T_{s}}{2 T_{f}}\right)}
\end{aligned}
$$

where $T_{d c}^{*}$ is the desired driving torque after filtering, $T_{s c}^{*}$ is the desired driving torque before filtering, $T_{s}$ is the control period, $T_{f}$ is the filtering time constant.

At the same time, the engine torque demand $T_{\text {eтсS }}$ should be smaller than the desired engine torque $T_{\text {eHEV }}$ which is calculated by an energy management system, otherwise engine torque will increase which is against the control aim of traction control systems to decrease driving torque.

To sum up, the engine torque demand $T_{\text {eTCS }}$ can be formulated as

$T_{\text {eTCS }}=\min \left(T_{d c}^{*}, T_{\text {eHEV }}\right)$

\subsection{Torque Dynamic Coordinate Control Strategy}

The aim of this torque dynamic coordinate control strategy is to achieve the desired driving torque rapidly and accurately. According to such requirements as fast response, accuracy, robustness and linear input/output transfer characteristics, a torque dynamic coordinate control strategy based on model matching 2-DOF control $^{[7]}$ is proposed as shown in Figure6

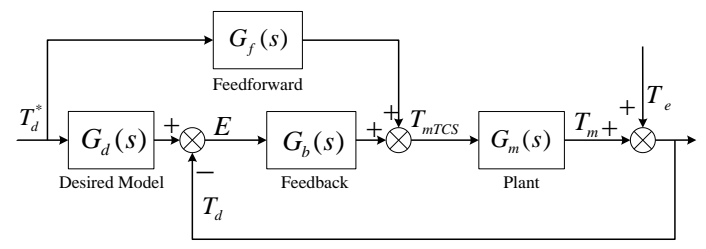

Figure6: Torque dynamic coordinate control strategy based on model matching 2-DOF control where $T_{d}$ is the actual driving total torque. In this strategy, the control plant is the motor model whose torque characteristics is

$T_{m}=\frac{1}{\tau_{m} s+1} T_{m T C S}=G_{m}(s) T_{m T C S}$

where $\tau_{m}$ is the really time constant of motor torque output.

The desired model is formulated as

$G_{d}(s)=\frac{1}{\tau_{d} s+1}$

where $\tau_{d}$ is the really time constant.

Then the feedforward transfer function can be calculated as

$G_{f}(s)=\frac{G_{d}(s)}{G_{m}(s)}=\frac{\tau_{m} s+1}{\tau_{d} s+1}$

Additionally, the PID control method is used in the feedback controller.

\section{Simulations and Analysis}

In order to evaluate the MHEVTCS, a simulation platform is built as shown in Figure7 including driver model, energy management controller model, MHEVTCS controller model, powertrain model and 15-DOF vehicle model.

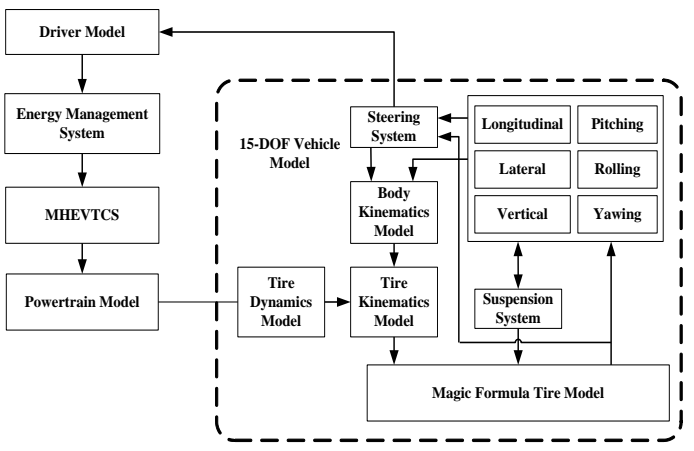

Figure7: Simulation platform

Two sets of MHEVTCS simulation are defined as shown in Table1.

Table1: Two sets of MHEVTCS simulation

\begin{tabular}{ccccc}
\hline & $\begin{array}{c}\text { Initial } \\
\text { Speed }\end{array}$ & $\begin{array}{c}\text { Gear } \\
\text { Ratio }\end{array}$ & $\begin{array}{c}\text { Adhesion } \\
\text { Coefficient }\end{array}$ & $\begin{array}{c}\text { Accelerator } \\
\text { Pedal }\end{array}$ \\
\hline S1 & $0 \mathrm{~m} / \mathrm{s}$ & 1 & $\begin{array}{c}\text { Left } 0.1 / \\
\text { Right } 0.8\end{array}$ & $100 \%$
\end{tabular}


In $\mathrm{S} 1$, the speeds of two slipping wheels are respectively controlled at target values after $0.5 \mathrm{~s}$ and $1 \mathrm{~s}$ with MHEVTCS as shown in Figure8. Acceleration performance of HEV increases $220 \%$ than without MHEVTCS. Vehicle speed comparison result shows that with ICETCS vehicle speed is only $3.1 \mathrm{~m} / \mathrm{s}$ at the end of simulation, and with MHEVTCS it achieves $3.43 \mathrm{~m} / \mathrm{s}$. Vehicle's acceleration performance is improved $11 \%$ by MHEVTCS than by ICETCS.
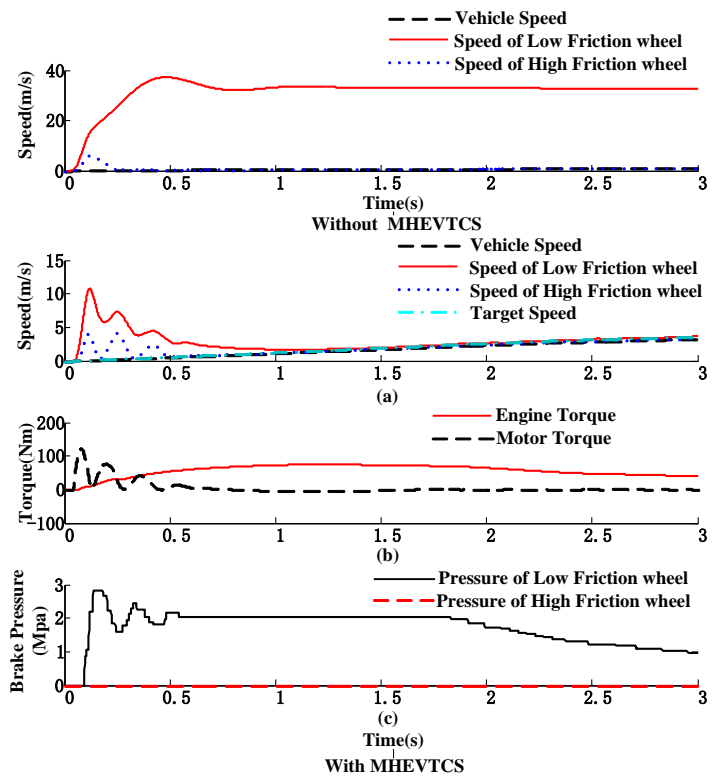

a) Control result

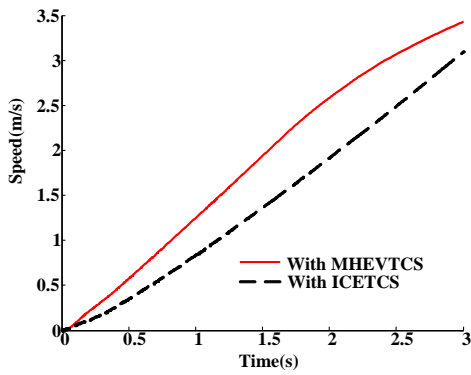

b) Speed comparison result

Figure8: Simulation results of S1

In S2, speed of the slipping wheel on low adhesion side road is controlled at target value after $0.4 \mathrm{~s}$ with MHEVTCS as shown in Figure9. Acceleration performance of $\mathrm{HEV}$ increases 5\% than without MHEVTCS. Lateral displacement comparison result shows that with ICETCS lateral displacement achieves $0.35 \mathrm{~m}$ at the end of simulation, and it is only $0.1 \mathrm{~m} / \mathrm{s}$ with MHEVTCS. Vehicle's stability performance is improved by MHEVTCS.

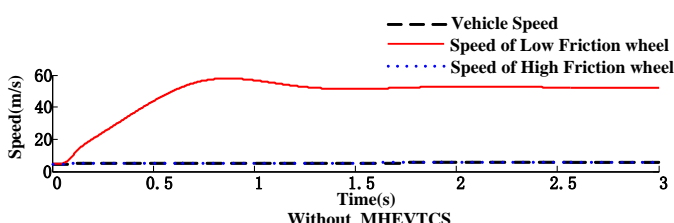
Without MHEVTCS
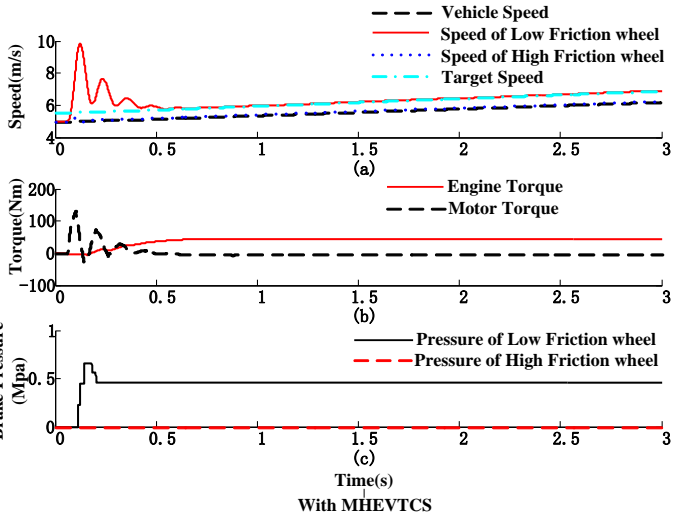

a) Control result

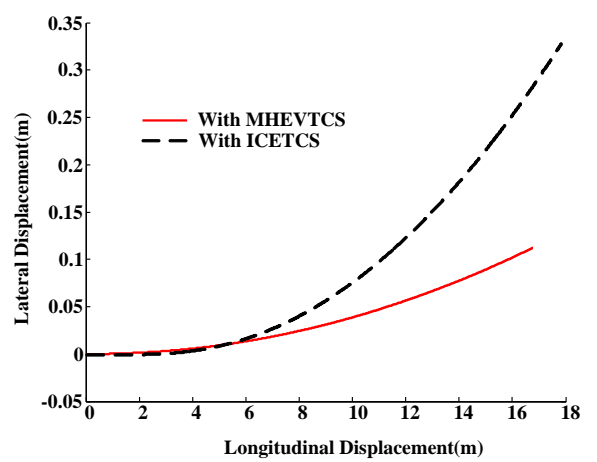

b) Lateral displacement comparison result

Figure9: Simulation results of S2

Furthermore, one hardware-in-loop test platform is built as shown in Figure10. 


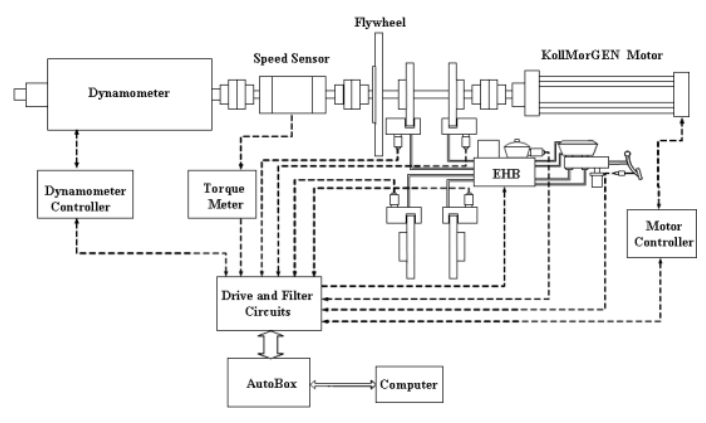

Figure10: Hardware-in-loop test platform

On this hardware-in-loop test platform, one set of tests is defined in Table2.

Table2: One set of hardware-in-loop tests

\begin{tabular}{ccccc}
\hline & $\begin{array}{c}\text { Initial } \\
\text { Speed }\end{array}$ & $\begin{array}{c}\text { Gear } \\
\text { Ratio }\end{array}$ & $\begin{array}{c}\text { Adhesion } \\
\text { Coefficient }\end{array}$ & $\begin{array}{c}\text { Accelerator } \\
\text { Pedal }\end{array}$ \\
\hline $\mathrm{S} 1$ & $0 \mathrm{~m} / \mathrm{s}$ & 1 & $\begin{array}{c}\text { Left } 0.1 / \\
\text { Right } 0.8\end{array}$ & $50 \%$ \\
\hline
\end{tabular}

Figure11 shows without MHEVTCS, the speed of low friction wheel achieves $18 \mathrm{~m} / \mathrm{s}$ and it is serious slipping. At the end of the test, vehicle speed is only $0.6 \mathrm{~m} / \mathrm{s}$. The vehicle can not start as normal.

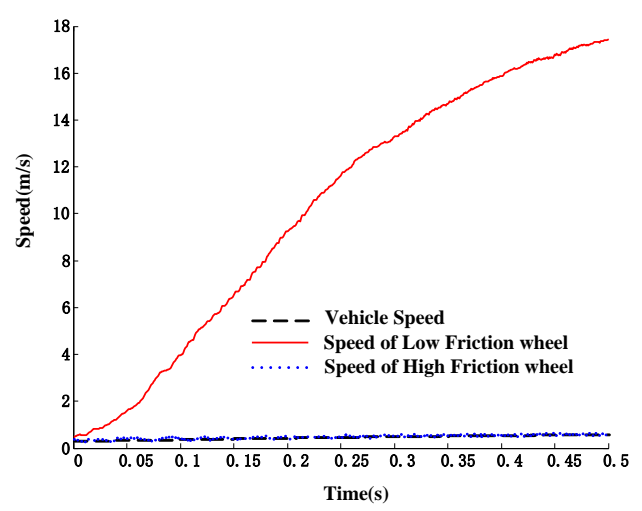

Figure11: Hardware-in-loop test result without MHEVTCS

Figure12 shows that the speed of slipping wheel on low adhesion side road is controlled at target value after $0.5 \mathrm{~s}$ with MHEVTCS. At the end of the test, vehicle speed achieves $4.8 \mathrm{~m} / \mathrm{s}$. Acceleration performance and starting ability of HEV are greatly improved by MHEVTCS.
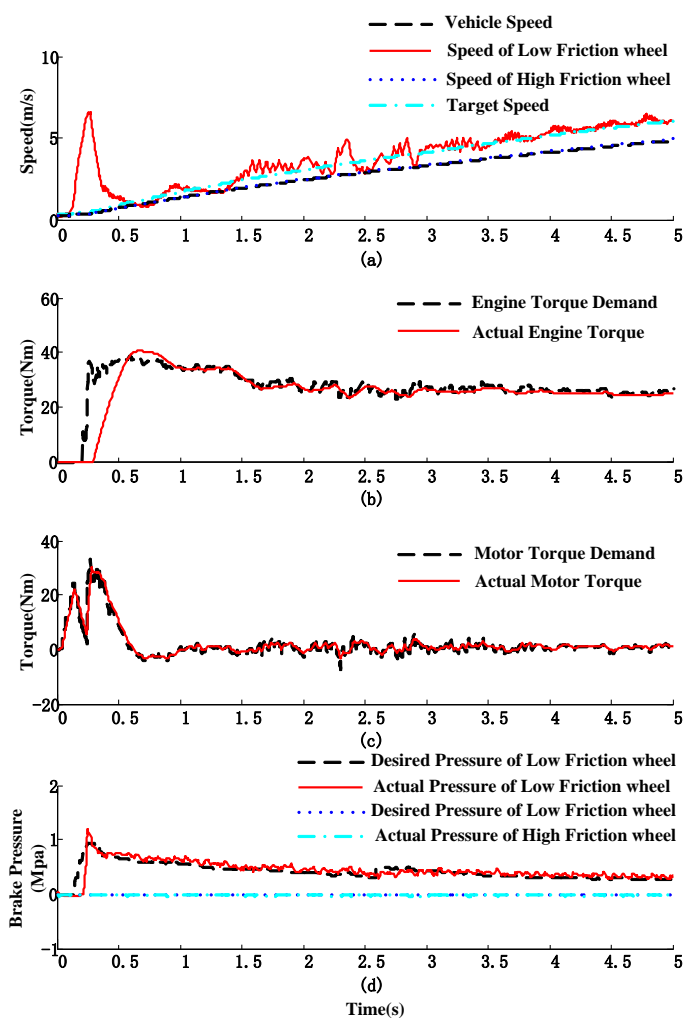

Figure12: Hardware-in-loop test result with

MHEVTCS

\section{Conclusions}

In this paper, a traction control method for an ISG hybrid electric vehicle is explored. The conclusions are followed:

1) Slipping wheels can be controlled by MHEVTCS quickly, accurately and smoothly.

2) Compared with conventional traction control systems, MHEVTCS improves vehicle's acceleration performance greatly without influencing its stability severely.

3) The dynamic coordinate control problem among engine, motor and hydraulic system is solved by MHEVTCS.

\section{Acknowledgments}

The authors greatly appreciate the support from the MOST (Ministry of Science and Technology) of China under the contract of No.2010DFA72760 and the Beijing Excellent Researchers Training Projects under 
the contract of 2011D009004000001.

\section{References}

[1] Kiyotaka Ise et. Al., The "Lexus" Traction Control (TRAC) System, SAE Paper No:900212

[2] Kazushi Hosomi et. Al., Development of Active-Traction Control Syste, SAE Paper No:2000-0101636

[3] Sakai S I et. Al., Advantage of electric motor for anti skid control of electric vehicle, Proceedings of the 9th International Conference on Power Electronics and Motion Control, Budapest, Hungary: IEEE, 2000: .45-51

[4] Sakai S I et. Al., Anti-skid control with motor in electric vehicle, Proceedings. 6th International Workshop on Advanced Motion Control, Nagoya, Japan: IEEE, 2000: 317-322.

[5] Li Shoubo et. Al., Traction Control of Hybrid Electric Vehicl,. Vehicle Power and Propulsion Conference, 2009, p.1536-1540

[6] Liao Chenglin et. Al., Modeling and simulation of traction control of hybrid electric vehicle, International Conference on Mechatronics and Automation, 2009, p.3252-3256

[7] Yasuhiro Oshiumi et. Al., Traction Force Control of Parallel Hybrid Electric Vehicle by Using Model Matching Controller, AVEC'98, 1998: 129-134.

\section{Authors}

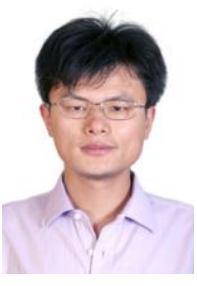

Feng Zhao received the Ph.D. degree from University of Science \& Technology Beijing, Beijing, China, in 2011, and the B.Tech. degree from Shandong University of Science \& Technology, Qingdao, China, in 2005. He is currently a postdoctor at the Department of Automotive Engineering, Tsinghua University, Beijing, China. His active research interests include Vehicle \& EV dynamics control and hybrid electric vehicle.

Yugong Luo received the Ph.D. degree from Tsinghua University, Beijing, China, in 2003, and the B.Tech. and M.S. degrees from Chongqing University, Chongqing, China, in 1996 and 1999 respectively. He is currently an Associate Professor with the Department of Automotive Engineering, Tsinghua University, Beijing, China. His active research interests include Vehicle \& EV dynamics and control, and Vehicle $\mathrm{NVH}$ analysis and control. He has authored over 30 journal papers and is a co-inventor on 15 patent applications. 
Keqiang $\mathrm{Li}$ received M.S. and Ph.D. degrees from Chongqing University in 1988 and 1995 respectively, and the B.Tech. degree from Tsinghua University in 1985 . He is currently a Professor with the Department of Automotive Engineering, Tsinghua University. His main areas of research interest include vehicle dynamics and control for driver assistance systems and hybrid electrical vehicles. He has authored over 90 papers and is a co-inventor of 12 patents in China and Japan. 